\title{
SECURITY CONSTRAINED OPTIMAL POWER DISPATCH USING ANT COLONY OPTIMIZATION ALGORITHM
}

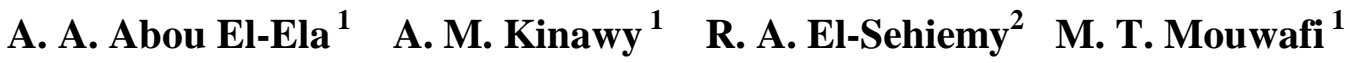 \\ 1) Electrical Engineering Department, Faculty of Engineering, Shebin El-Kom, \\ Minoufiya University, Egypt \\ 2) Electrical Engineering Department, Faculty of Engineering, \\ Kafrelsehiekh University, Egypt
}

\begin{abstract}
This paper proposes a procedure for solving a security constrained optimal power dispatch (SCOPD) problem under normal and emergency conditions using ant colony optimization (ACO) algorithm. The objective function is to minimize the non-linear generation cost function by optimizing the control variables of the generators real power under equality and inequality constraints.

The ACO algorithm is applied to 5- bus system, the IEEE standard 14-bus and 30-bus systems. In addition, an application of the proposed algorithm to a real power system at the west Delta network (WDN) as a part of the Unified Egyptian Network (UEN) considering the valve-points effects has been demonstrated. The results obtained are compared with those obtained using a conventional linear programming (LP), the fuzzy linear programming (FLP) technique and genetic algorithm (GA). Simulation results show that the proposed ACO algorithm for the SCOPD is more accurate and efficient, especially with increasing the system size.

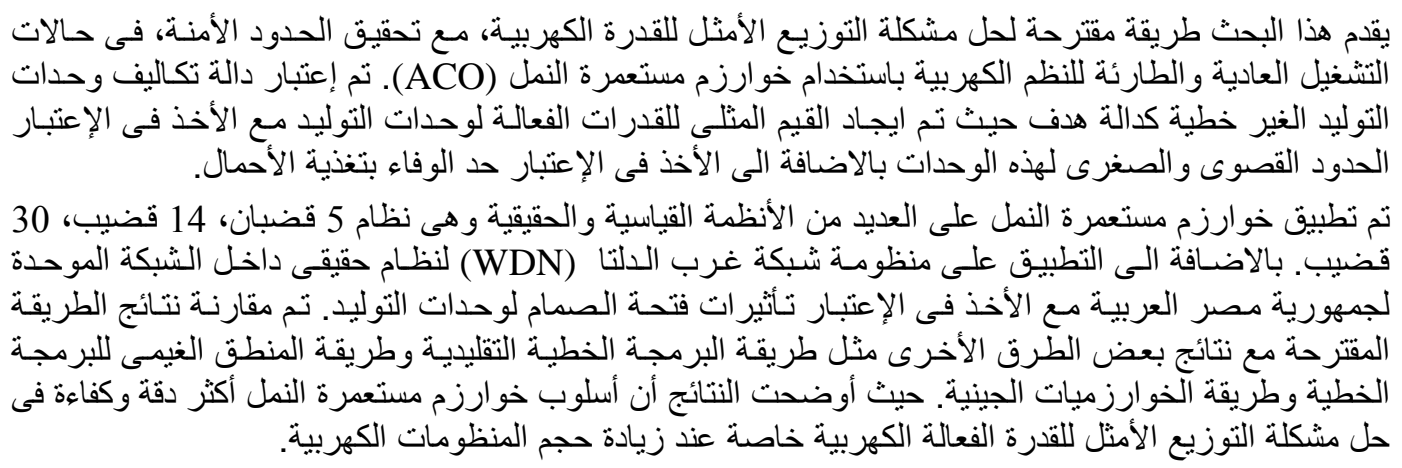

Keywords: Optimal power dispatch, Security, Ant colony optimization algorithm, Valve-points

\section{INTRODUCTION}

Security constrained optimal power dispatch (SCOPD) is one of the optimization problems in power systems that optimally allocate the power demand among committed generators in the most economical manner while satisfying system security constraints. The problem becomes more complicated due to the non-linear nature of the objective function and constraints of real life problems. The objective function may be convex or nonconvex based on the characteristic of the supply. The next paragraph presents the different optimization methods that can be used for solving the economic dispatch (ED) problem.

Madrigal and Quintana [1] presented an analytical solution to the classic economic dispatch problem using duality theory to derive an expression to compute both the exact primal (dispatches) and dual (marginal cost) solution to the problem without the need for numerical iterative optimization algorithms. No conflict of interest is caused if the (ED) model is used as an optimization-based electricity auction. C. Chen and N. Chen [2] solved the economic dispatch problem considering transmission capacity constraints using direct search method (DSM) to handle a number of inequality and equality constraints and units with any kind of fuel cost functions. For improving the performance of direct search procedure, a novel strategy with multilevel convergence is incorporated in the DSM to minimize the number of total iterations in the searching process. Pathom et al. [3] presented a methodology for solving the dynamic economic dispatch (DED) problem using evolutionary programming (EP) combined with sequential quadratic programming (SQP) that consists of two 
parts. The first part employed the property of EP which can provide a near global search region at the beginning. When the specified termination criteria of $\mathrm{EP}$ are reached, the local search SQP is applied to tune the control variables to obtain the final optimal solution. Xia and Song [4] proposed a novel approach based on the analysis of the process of solution of ED problem by Lagrangian Relaxation, called dynamic queuing (DQ) algorithm. Yan and Quintana [5] presented an improving an interiorpoint based OPF by a predictor-corrector primal-dual log-barrier (PCPDLB) method as a sequence of linearized sub-problems. Jabr et al. [6] presented a homogeneous interior point (HIP) method for the ED problem by approximating the network constraints through the DC load flow, and the transmission losses through the B-matrix loss formula. Liu et al. [7] analyzed the mixed integer OPF based on the interior point cutting plane method (IPCPM). Also, they presented a new base identification method based on the improvement of IPCPM to solve the problems of degenerate solutions and convex combination solutions that depends on the difference between nonzero element number of optimal solution and rank of coefficient matrix. Vlaisavljevic et al. [8] demonstrated the feasibility of using a fuzzy expert system, based on interactive fuzzy linear programming (FLP) to optimal power system rescheduling problem, incorporating the preventive redispatch. Their aim was to explore the feasibility of creating an intelligent power system rescheduling system. Pathom et al. [9] proposed the fuzzyoptimization approach for solving the (DED) under an uncertain deregulated power system. Amjady and Nasiri- Red [10] presented a RCGA with arithmeticaverage-bound crossover (AABX) and hybrid mutation (HM) to solve the nonconvex ED problem. Through few recent years, ACO algorithms are employed to solve optimization problems in different fields with more accurate and efficiently solution compared with conventional and other modern optimization algorithms.

In this paper, an approach is proposed to solve the nonsmooth ED problem using the ACO algorithm, which based on the behavior of real ants for searching the shortest route between the colony and the souce of food based on the indirect communication media, called pheromone. The minimization of the total fuel generation costs is considered as an objective function with equality and inequality constraints. This paper is organized as; Section II formulates the considered ED problem that shows the objective function and constraints. Section III is divided into two parts. Part 1 shows the description of real ants for searching the shortest route between nest and food source. Part 2 introduces the mathematical model of ACO algorithm that based on the probabilistic transition rule and the pheromone updates. In Section IV, the ACO algorithm is implemented to the ED problem. Section V contains the results obtained by the application of the ACO algorithm to the 5-bus, IEEE-14 and 30 bus test systems under normal and emergency conditions which are compared with the results obtained using the conventional LP, fuzzy linear programming (FLP) and GA. Also, the ACO algorithm is applied to a real power system at the WDN system as a part of the Unified Egyptian Network (UEN). The results show that, the proposed algorithm is more accurate and efficient in solving the ED problem.

\section{PROBLEM FORMULATION}

The SCOPD problem can express as a constrained optimization problem as:

$$
\begin{array}{ll}
\operatorname{Min} f(x) \\
\text { s. t.: } & g(x)=0 \\
& h(x) \leq 0
\end{array}
$$

where, $f(x)$ is the objective function such as generators fuel costs, transmission line losses,...etc, $g(x)$ represents the equality constraints, $h(x)$ represents the inequality constraints, and $x$ is the vector of the control variables that may be generator real power outputs, generator voltages, switchable reactive power and transformer tap setting. In this paper, the objective function is the non-linear fuel cost of generators with the valve-point effects that appears in a rectified sinusoidal function introduce ripples in the heat-rate curves, that's a function in the generator real power output, which are defined, as:

$$
\begin{aligned}
\operatorname{MinF}_{t}=\sum_{i=1}^{N G} f_{i}\left(P G_{i}\right) & =\sum_{i=1}^{N G} a_{i}+b_{i} P G_{i}+c_{i} P G_{i}{ }^{2} \\
& +\left|e_{i} \times \sin \left(f_{i} \times\left(P G_{i}^{\text {min }}-P G_{i}\right)\right)\right|
\end{aligned}
$$

where,

$F_{t}$ : is the non-linear objective function of power generation cost.

$a_{i}, b_{i}$ and $c_{i}$ are the coefficients of power generation cost function.

$e_{i}$ and $f_{i}$ are the fuel cost coefficients of the $i$ th unit with valve-points effects.

$N G$ : is the number of generation buses.

The objective function (3) is subjected to the following constraints:

\section{a) Equality constraints}

- Power balance constraint

The generators real power output should be equal to the total load demand and transmission line losses as:

$$
\sum_{i=1}^{N G} P G_{i}=\sum_{j=1}^{N L} P D_{j}+P_{\text {losses }}
$$


where, $P G_{i}$ is the power generation at bus $i, P D_{j}$ is the load demand at load bus $j, N L$ is the number of load buses, and $P_{\text {losses }}$ is the total power losses in the system.

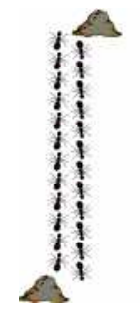

a.

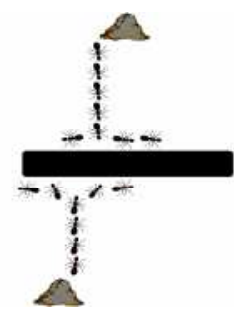

b.

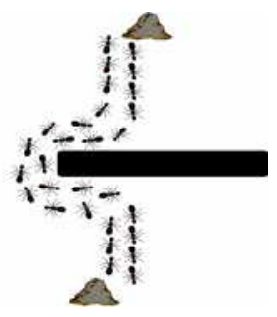

c.
Fig. 1 Illustration of real ant behavior

\section{b) Inequality constraints}

- The generator real power output must be within the feasible limits as:

$$
P G_{i}^{\min } \leq P G_{i} \leq P G_{i}^{\max }
$$

where, $P G_{i}^{\max }$ and $P G_{i}^{\min }$ are the maximum and minimum limits of power generation at bus $i$, respectively.

- Power flow constraints

The power flow in each transmission must be less than the maximum limit of power flow in that line as:

$$
\left|P F_{k}\right|=\left|D_{k, i} P G_{i}\right| \prec P F_{k}^{\max }
$$

where, $P F_{k}$ is the power flow in line $k, P F_{k}{ }^{\text {max }}$ is the maximum power flow in line $k$ and $D_{k, i}$ is the sensitivity parameters of the power flows related to the power generations.

\section{ACO ALGORITHM}

ACO algorithms were first proposed by Dorigo [11] and his related future work [12-13].

\subsection{Description of real ants}

ACO algorithms are based on the behavior of real ants that are members of a family of social insects. However, a group of explorer ants leave the colony for finding the food source in a randomly directions where they marked their routes by laying a chemical substance on the ground. Other ants attractive to the route that has the largest amount of pheromone that decays with time. So that, a shorter route will be found that has a largest amount of pheromone than a longer route. Hence, they are found the shortest route between the nest and food source by indirect communication media that called pheromone that laid on the ground as a guide for another ants. Fig. 1 shows how the real ants can find the shortest path between nest or colony and food source. In Fig. 1-a, there are no obstacles between nest and food source. However, the shortest route is the straight line. If an obstacle is located on the route of ants to become two routes around the obstacle, some of ants choose the left side around the obstacle and the other will choose the right side as shown in Fig. 1-b. The pheromone laid on the left side will be concentrated than right side of obstacle because ants in the shortest path takes minimum time in leaving and returning for nest where they moves in the same speed. So, they will be laid a largest amount of pheromone than other ants on the other route. While, the other ants attractive to the shortest route. Hence, all ants in the colony will take the shortest route around the obstacle as shown in Fig. 1-c.

\section{2. Mathematical Model of ACO Algorithm}

A random amount of pheromone is deposited in each rout after each ant completes its tour, anther antes attract to the shortest route according to the probabilistic transition rule that depends on the amount of pheromone deposited and a heuristic guide function as equal to the inverse of the distance between beginning and ending of each route. The probabilistic transition rule of ant $k$ to go from city $i$ to city $j$ can be expressed as in Traveling Salesman Problem (TSP) [13] as:

$$
P_{i j}^{k}(t)=\frac{\left[\tau_{i j}(t)\right]^{\alpha}\left[\eta_{i j}(t)\right]^{\beta}}{\sum_{q}\left[\tau_{i q}(t)\right]^{\alpha}\left[\eta_{i q}(t)\right]^{\beta}} ; j, q \in N_{i}^{k}
$$

where, $\tau_{i j}$ is the pheromone trail deposited between city $i$ and $j$ by ant $k$; $\eta_{i j}$ is the visibility or sight and equal to the inverse of the distance or the transition cost between city $i$ and $j\left(\eta_{i j}=1 / \mathrm{d}_{\mathrm{ij}}\right) . \alpha$ and $\beta$ are two parameters that influence the relative weight of pheromone trail and heuristic guide function, respectively. If $\alpha=0$, the closest cities are more likely to be selected that corresponding to a classical greedy algorithm. On the contrary, if $\beta=0$, the probability will be depend on the pheromone trial only. These two parameters should be tuned with each other, Dorigo in [11] found experimentally the good values of $\alpha$ and $\beta$ are 1 and 5, respectively, $q$ is the cities that will be visited after city $i$. While, $N_{r}{ }^{k}$ is a tabu list in memory of ant that recodes the cities are visited to avoid stagnations. After each tour is completed, a local pheromone update is determined by each ant depending on the route of each ant as in equation (8). After all ants attractive to the shortest route, a global pheromone update is considered to show the influence of the new addition deposits by the other ants that attractive to the best tour as shown in equation (9).

$$
\begin{aligned}
& \tau_{i j}(t+1)=(1-\rho) \tau_{i j}(t)+\rho \tau_{\circ} \\
& \tau_{i j}(t+1)=(1-\rho) \tau_{i j}(t)+\varepsilon \Delta \tau_{i j}(t)
\end{aligned}
$$

where, $\tau_{i j}(t+1)$ is the pheromone after one tour or iteration. $\rho$ is the pheromone evaporation constant equals to 0.5 as a good value by Dorigo in [11]. $\varepsilon$ is the elite path weighting constant. $\tau_{o}=1 / d_{i j}$ is the incremental value of pheromone of each ant. While, $\Delta \tau_{i j}$ is the amount of pheromone for elite path as: 


$$
\Delta \tau_{i j}(t)=1 / d_{\text {best }}
$$

where, $d_{\text {best }}$ is the shortest tour distance found as in TSP.

\section{ACO ALGORITHM FOR SCOPD PROBLEM}

ACO algorithm is applied to solve the SCOPD problem as an optimization technique with equality and inequality constraints where artificial ants travels in search space to find the shortest route that having the strongest pheromone trail and a minimum cost function. Our objective in this paper is to minimize the total cost of generation real output power as described in (3) with equality constraint (4) and inequality constraints in (5) and (6). So, heuristic guide function is the inverse of the individual cost of each ant that positioned in the reasonable limit of the control variable to the visibility of each ant. While, heuristic guide function of the problem is the inverse of the total costs at iteration $t+1$ as:

$$
\eta(t+1)=1 / \sum_{i=1}^{N G} f_{i}\left(P G_{i}\right)
$$

In GA, a chromosome is subdivided into genes, each gene represents a variable, consists of a binary string with length that depends on the boundary of the corresponding variable. While, in particle swarm optimization (PSO), a swarm consists of particles or populations that corresponding to the control variables. In ACO algorithm, a search space creates with dimensions of stages on number of control variables and states or the randomly distributed values of control variables with in a reasonable threshold. Artificial ants leaves colony to search randomly in the search space based on the probability in (7) to complete a tour matrix that consists of the positions of ants with the same dimension of the search space. Then, tour matrix is applied on the objective function to find a heuristic guide function to find the best solution and update local and global pheromone to begin a next iteration. System parameters are adjusted by trail and error to find the best values of theses parameters.

The ACO algorithm can be applied to solve the SCOPD problem using the following steps:

\section{Step 1: Initialization}

Insert the lower and upper boundaries of each control variable $\left(\mathrm{PG}^{\mathrm{min}}, \mathrm{PG}^{\mathrm{max}}\right)$, system parameters and create a search space with a dimensions of number of control variables (PG) and the length of randomly distributed values with the same dimension of the initial pheromone that contains elements with very small equal values to give all ants with the same chance of searching.

\section{Step 2: Provide first position}

Each ant is positioned on the initial state randomly with in the reasonable range of each control variable in a search space with one ant in each control variable in the length of randomly distributed values.

\section{Step 3: Transition rule}

Each ant decide to visit a next position in the range of other control variables according to the probability transition rule in equation (7) that depends on the amount of pheromone deposited and the visibility that is the inverse of objective function (11). Where, the effect of pheromone and visibility on each other depends on the two parameters $\alpha$ and $\beta$.

\section{Step 4: Local pheromone updating}

Local updating pheromone is different from ant to other because each ant takes a different route. The initial pheromone of each ant is locally updated as in equation (8).

\section{Step 5: Fitness function}

After all ants attractive to the shortest path that having a strongest pheromone, the best solution of the objective function is obtained

\section{Step 6: Global pheromone updating}

Amount of pheromone on the best tour becomes the strongest due to attractive of ants for this path. Moreover, the pheromone on the other paths is evaporated in time.

\section{Step 7: Program termination}

The program will be terminated when the maximum iteration is reached or the best solution is obtained without the ants stagnations.

\section{APPLICATIONS}

\section{1. Test Systems}

Three standard test systems and a real power system are used to study the proposed technique for SCOPD using an ACO algorithm. The first test system contains 5 buses and 7 transmission lines [14]. The second system is IEEE 14-bus test system [15], while the third is IEEE 30-bus test system [15]. The critical lines are number 1 in all test systems. The maximum power flow ratings of these critical lines are equal to 45,150 and $65 \mathrm{MW}$ for the three systems, respectively. However the ratings of the other lines in the three systems are below their security limits. The results that obtained are compared with those obtained in a previous work using a conventional linear programming (LP), the fuzzy linear programming (FLP) and the genetic algorithm (GA) technique [14]. The real power system is the WDN system (Fig. 2) as a part of the Unified Egyptian Network (UEN) [16]. The best values of ACO algorithm parameters are $\alpha=1, \beta=8$, $\rho=0.5$ and $\varepsilon=5$

Two different operation conditions are considered to obtain the SCOPD, which are normal and emergency conditions. 
The emergency conditions that may occur in the three test systems are:

\section{2. Results and Comments}

The results are obtained using ACO algorithm that processed using MatLab code version 7.1 that setup on a Pentium 4, 3.0 GHz PC, 0.99 GB of ram.

\subsubsection{Normal conditions}

Tables 1, 2 and 3 show a comparison between the results obtained using ACO algorithm and the previous results using conventional LP, the FLP and GA [14]. In theses tables, the ACO algorithm has the minimum generation cost compared with other techniques.

Table 4 shows a comparison between the results obtained using ACO algorithm and the results using conventional LP, the FLP and GA for WDN system with valve-point effects are taken into account. In this table, the ACO algorithm has the minimum generation cost compared with other techniques. The computation time using ACO algorithm dependent on the system size and related to the number of
a) Sudden increase in load demand.
b) Unexpected outage of one line.
c) Unexpected outage of units inside the generation plant.

control variables in tables $1-4$, the computation time is $2.1,2.25,2.28$ and 2.344 corresponding to 14,5 , 30 and WDN systems that have 2, 3, 6 and 8 number of control variables.

Table 1 Comparison of various optimization methods for 5-bus system (load=185 MW)

\begin{tabular}{lllll}
\hline & \multicolumn{1}{c}{ LP } & \multicolumn{1}{c}{ FLP } & \multicolumn{1}{c}{ GA } & \multicolumn{1}{c}{ ACO } \\
\hline PG1 & 90.2 & 78.9 & 90.2 & 90.15 \\
PG2 & 34.8 & 61.7 & 35.8 & 35.17 \\
PG5 & 60 & 44.4 & 59 & 59.68 \\
PF1 & 45 & 34.6 & 44.98 & 44.94 \\
Cost. $\mathbf{\$} / \mathbf{h r}$ & $\mathbf{3 8 0 . 7}$ & $\mathbf{3 9 1 . 7}$ & $\mathbf{3 7 4 . 1}$ & $\mathbf{3 7 3 . 8}$ \\
Time. Sec & 0.55 & 0.66 & 0.99 & 2.25 \\
\hline
\end{tabular}

Table 2 Comparison of various optimization methods for 14-bus system (load=260 MW)

\begin{tabular}{|c|c|c|c|c|}
\hline & LP & FLP & GA & $\mathrm{ACO}$ \\
\hline PG1 & 208.1 & 196.8 & 208.1 & 208.01 \\
\hline PG2 & 51.86 & 63.2 & 51.9 & 51.99 \\
\hline PF1 & 150 & 140.4 & 149.96 & 149.92 \\
\hline Cost. $\$ / h r$ & 958.1 & 961.4 & 767.5 & 763.4 \\
\hline Time. Sec & 0.5 & 0.72 & 1.1 & 2.1 \\
\hline
\end{tabular}

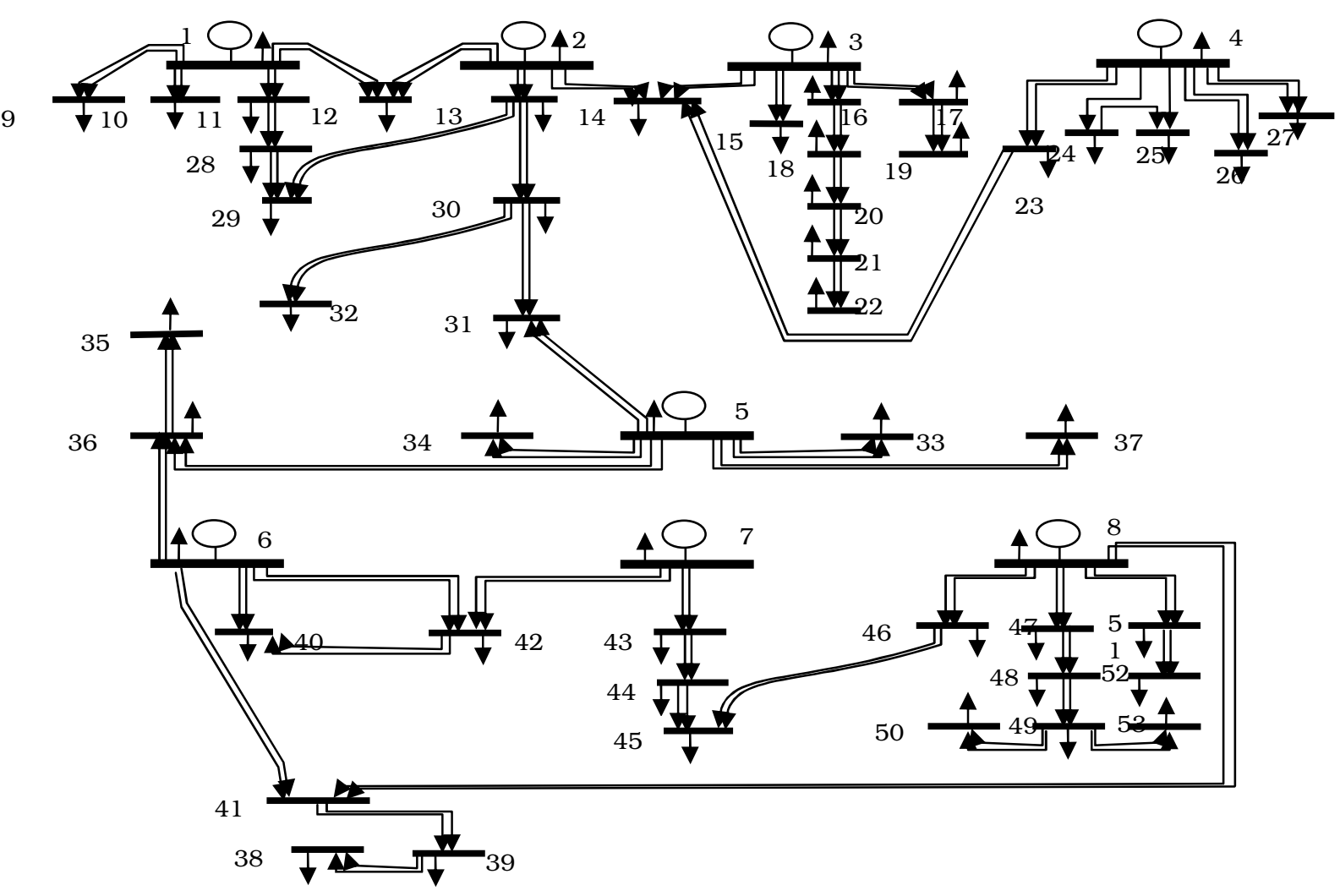

Fig. 2 Single line diagram of the WDN system 
A. A. Abou El-Ela, A. M. Kinawy, R. A. El-Sehiemy, M. T. Mouwafi, "Security Constrained Optimal Power ..."

Table 3 Comparison of various optimization methods for 30-bus system (load=220 MW)

\begin{tabular}{lllll}
\hline & LP & FLP & GA & ACO \\
\hline PG1 & 10 & 52 & 49.1 & 59.73 \\
PG2 & 80 & 62.3 & 65.6 & 55.89 \\
PG3 & 39.4 & 28.9 & 21 & 40.81 \\
PG4 & 10 & 16 & 23.7 & 17.5 \\
PG5 & 30 & 25.5 & 16.5 & 27.12 \\
PG6 & 50.6 & 35.3 & 44.1 & 18.95 \\
PF1 & -0.251 & 30.94 & 28.07 & 37.75 \\
Cost. $\$$ /hr & $\mathbf{8 7 1 . 9 3}$ & $\mathbf{8 7 9 . 2 2}$ & $\mathbf{6 8 5 . 1 3}$ & $\mathbf{6 5 4 . 9}$ \\
Time. Sec & 0.6 & 1.1 & 1.75 & 2.28 \\
\hline
\end{tabular}

\section{2.2 Emergency Conditions}

\section{- Sudden increase in the load demand}

Tables 5 and 6 show the SCOPD using the ACO algorithm for different loading conditions for the 5bus and 14- bus test systems.

In these tables, the power flows in the critical lines are kept within their limits, and the generation costs are increased according to the increasing of the load demand.

Table 7 shows a comparison between the results obtained using ACO algorithm and GA for a real power system for different loading conditions. In this table, the ACO algorithm has the minimum generation cost compared with GA.

\section{- Unexpected outage of transmission line}

Tables 8 and 9 show the SCOPD computed using the ACO algorithm for different lines outage compared with the load flow (LF) using the NewtonRaphson method for 5-bus and 14-bus test systems. In these tables, overflows in the critical lines are removed using the ACO algorithm.

Table 10 shows the SCOPD computed using the ACO algorithm for different lines outage compared with the load flow (LF) using the Newton-Raphson method for real power system. The power flows in all lines are kept within their permissible limits.
Table 4 Comparison of various optimization methods for the west delta network system

$($ load=890 MW)

\begin{tabular}{lllll}
\hline & LP & \multicolumn{1}{c}{ FLP } & \multicolumn{1}{c}{ GA } & ACO \\
\hline PG1 & 10 & 107.6704 & 70 & 62.218 \\
PG2 & 10 & 123.2776 & 84.5 & 90.853 \\
PG3 & 10 & 116.9019 & 81.2 & 83.74 \\
PG4 & 250 & 104.6504 & 130.1 & 131.68 \\
PG5 & 339.75 & 140.9867 & 192.5 & 170.08 \\
PG6 & 250 & 74.3218 & 220.1 & 221.91 \\
PG7 & 10 & 104.6519 & 56.2 & 66.42 \\
PG8 & 10 & 117.2894 & 55.3 & 63.026 \\
PF34 & 32.417 & 12.88 & 18.192 & 4.325 \\
PF40 & 46.248 & 26.636 & 46.603 & 12.522 \\
PF50 & 4.5307 & 12.535 & 9.1532 & 16.665 \\
PF76 & 15.734 & 12.298 & 14.918 & 16.745 \\
Cost. $\mathbf{\text { /hr }}$ & $\mathbf{3 1 4 9 . 3}$ & $\mathbf{3 8 9 0 . 4}$ & $\mathbf{2 9 0 9 . 8}$ & $\mathbf{2 9 0 3 . 9}$ \\
Time. Sec & 0.375 & 0.594 & 2.937 & 2.344 \\
\hline
\end{tabular}

Where, the maximum power flow in lines $34,40,50$ and 76 are 200, 150, 200 and $200 \mathrm{MW}$, respectively.

Table 5 SCOPD solution using ACO algorithm for different loading conditions for 5-bus system

\begin{tabular}{lllllll}
\hline $\begin{array}{c}\text { Load } \\
\text { (MW) }\end{array}$ & $\mathbf{1 5 0}$ & $\mathbf{1 7 0}$ & $\mathbf{1 8 5}$ & $\mathbf{2 0 0}$ & $\mathbf{2 2 0}$ & $\mathbf{2 3 0}$ \\
\hline PG1 & 82.74 & 86.87 & 90.15 & 92.36 & 92.63 & 93.06 \\
PG2 & 14.51 & 27.93 & 35.17 & 49.76 & 69.83 & 79.67 \\
PG3 & 52.75 & 55.2 & 59.68 & 57.88 & 57.54 & 57.27 \\
PF1 & 44.83 & 44.86 & 44.94 & 43.92 & 40.397 & 38.88 \\
Cost (\$/hr ) & $\mathbf{2 9 5 . 1 2}$ & $\mathbf{3 4 0 . 0 1}$ & $\mathbf{3 7 3 . 8}$ & $\mathbf{4 0 9 . 6 2 6}$ & $\mathbf{4 6 0 . 2 4}$ & $\mathbf{4 8 5 . 9 2}$ \\
\hline
\end{tabular}

Table 6 SCOPD solution using ACO algorithm for different loading conditions for 14-bus system

\begin{tabular}{lllllll}
\hline Load (MW) & $\mathbf{2 2 0}$ & $\mathbf{2 4 0}$ & $\mathbf{2 6 0}$ & $\mathbf{2 7 0}$ & $\mathbf{2 8 0}$ & $\mathbf{2 8 5}$ \\
\hline PG1 & 203.47 & 206.31 & 208.01 & 209.13 & 210.84 & 210.74 \\
PG2 & 16.53 & 33.69 & 51.99 & 60.87 & 69.16 & 74.26 \\
PF1 & 149.1 & 149.52 & 149.92 & 149.94 & 149.97 & 149.98 \\
Cost (\$/hr ) & $\mathbf{6 1 5 . 7 5}$ & $\mathbf{6 8 9 . 0 6}$ & $\mathbf{7 6 3 . 4}$ & $\mathbf{8 0 7 . 9 8}$ & $\mathbf{8 5 0 . 3}$ & $\mathbf{8 7 2 . 5 3}$ \\
\hline
\end{tabular}

Table 7 SCOPD solution using ACO algorithm and GA for different loading conditions for the west delta system

\begin{tabular}{|c|cc|c|c|c|c|c|c|c|c|}
\hline Load(MW) & \multicolumn{2}{|c|}{$\mathbf{7 0 0}$} & \multicolumn{2}{|c|}{$\mathbf{8 0 0}$} & \multicolumn{2}{c|}{$\mathbf{8 9 0}$} & \multicolumn{2}{c|}{$\mathbf{1 0 0 0}$} & \multicolumn{1}{|c|}{$\mathbf{1 1 0 0}$} \\
\hline Algorithm & GA & ACO & GA & ACO & GA & ACO & GA & ACO & GA & ACO \\
\hline PG1 & 70 & 55.588 & 70 & 59.732 & 70 & 62.218 & 75.6 & 72.165 & 83.1 & 84.598 \\
PG2 & 69.9 & 71.789 & 88.5 & 82.189 & 84.5 & 90.853 & 130 & 106.3 & 130 & 125.51 \\
PG3 & 73.3 & 72.679 & 70 & 76.366 & 81.2 & 83.74 & 113.8 & 94.801 & 114.9 & 114.71 \\
PG4 & 100 & 106.99 & 100 & 124.98 & 130.1 & 131.68 & 129.9 & 137.98 & 160.2 & 159.5 \\
PG5 & 101.2 & 101.6 & 161.7 & 146.11 & 192.5 & 170.08 & 186.6 & 189.77 & 197 & 198 \\
PG6 & 197.5 & 201.19 & 210.6 & 213.43 & 220.1 & 221.91 & 220 & 241.62 & 249.8 & 249.88 \\
PG7 & 40 & 39.13 & 40 & 51.702 & 56.2 & 66.42 & 69.3 & 77.459 & 82.8 & 83.146 \\
PG8 & 48.1 & 50.967 & 59.1 & 45.413 & 55.3 & 63.026 & 74.8 & 79.832 & 82.2 & 84.583 \\
PF34 & 9.4366 & 9.5258 & 15.2154 & 13.7417 & 18.192 & 15.9839 & 17.4488 & 17.8139 & 18.4086 & 18.5239 \\
PF40 & 42.0418 & 42.0556 & 43.8706 & 44.787 & 46.603 & 47.3803 & 48.9796 & 52.1595 & 55.3235 & 55.3071 \\
PF50 & 2.8701 & 5.0786 & 6.2583 & 5.7262 & 9.1532 & 7.148 & 8.8535 & 8.5202 & 9.5783 & 9.6635 \\
PF76 & 8.974 & 10.4068 & 11.7094 & 11.9146 & 14.918 & 13.2523 & 14.1096 & 14.7584 & 15.9008 & 16.0192 \\
Cost. \$/hr & $\mathbf{2 1 7 3}$ & $\mathbf{2 1 6 9 . 3}$ & $\mathbf{2 5 4 9 . 1}$ & $\mathbf{2 5 4 4 . 6}$ & $\mathbf{2 9 0 9 . 8}$ & $\mathbf{2 9 0 3 . 9}$ & $\mathbf{3 3 6 7 . 9}$ & $\mathbf{3 3 6 5}$ & $\mathbf{3 7 9 9 . 4}$ & $\mathbf{3 7 9 8 . 9}$ \\
Time. Sec. & 3.063 & 2.219 & 2.891 & 2.266 & 2.937 & 2.344 & 2.922 & 2.343 & 3.031 & 3.462 \\
\hline
\end{tabular}


Table 8 SCOPD solution for different line outage for 5-bus test system (load=185 MW)

\begin{tabular}{lllllll}
\hline Line Outage & $\mathbf{1}$ & \multicolumn{2}{l}{$\mathbf{2}$} & \multicolumn{3}{l}{$\mathbf{6}$} \\
\hline Algorithm & LF & ACO & LF & ACO & LF & ACO \\
\hline PG1 & 90.15 & 59.98 & 90.15 & 59.89 & 90.15 & 85.61 \\
PG2 & 35.17 & 69.10 & 35.17 & 69.24 & 35.17 & 40.12 \\
PG3 & 59.68 & 55.92 & 59.68 & 55.87 & 59.68 & 59.27 \\
PF1 & ---- & ---- & $77.15^{*}$ & 44.78 & $49.46^{*}$ & 44.23 \\
PF2 & $73.557^{*}$ & 44.735 & ---- & ---- & 31.65 & 26.35 \\
\hline
\end{tabular}

* Denotes an overflow in transmission line

Where, the maximum power flow in line 2 is $45 \mathrm{MW}$

Table 9 SCOPD solution for different line outage for 14-bus test system (load=260 MW)

\begin{tabular}{lllllll}
\hline Line Outage & \multicolumn{1}{c}{$\mathbf{6}$} & \multicolumn{1}{c}{$\mathbf{6}$} & \multicolumn{3}{c}{$\mathbf{1 0}$} \\
\hline Algorithm & LF & ACO & LF & ACO & LF & ACO \\
\hline PG1 & 208.01 & 185.63 & 208.01 & 203.45 & 208.01 & 204.28 \\
PG2 & 51.99 & 74.37 & 51.99 & 56.55 & 51.99 & 55.72 \\
PF1 & $166.49 *$ & 149.38 & $151.99 *$ & 148.46 & $151.45 *$ & 149.41 \\
PF7 & ---- & ---- & 47.016 & 28.204 & 98.379 & 94.251
\end{tabular}

* Denotes an overflow in transmission line

Where, the maximum power flow in line 7 is $100 \mathrm{MW}$

Table 10 SCOPD solution for different line outage for the west delta network system (load=890 MW)

\begin{tabular}{lllllll}
\hline Line Outage & $\mathbf{3 4}$ & \multicolumn{3}{l}{$\mathbf{4 0}$} & \multicolumn{3}{c}{$\mathbf{5 0}$} \\
\hline Technique & LF & ACO & LF & ACO & LF & ACO \\
\hline PG1 & 62.218 & 64.559 & 62.218 & 63.315 & 62.218 & 64.973 \\
PG2 & 90.853 & 91.079 & 90.853 & 90.981 & 90.853 & 91.177 \\
PG3 & 83.74 & 84.212 & 83.74 & 85.081 & 83.74 & 85.818 \\
PG4 & 131.68 & 131.5 & 131.68 & 131.8 & 131.68 & 132.1 \\
PG5 & 170.08 & 174.28 & 170.08 & 175.99 & 170.08 & 174.28 \\
PG6 & 221.91 & 224.71 & 221.91 & 223.77 & 221.91 & 221.88 \\
PG7 & 66.42 & 63.92 & 66.42 & 65.227 & 66.42 & 64.92 \\
PG8 & 63.026 & 55.668 & 63.026 & 53.764 & 63.026 & 54.775 \\
PF34 & --- & ---- & 79.492 & 16.234 & 82.231 & 16.408 \\
PF40 & 40.739 & 47.209 & ---- & --- & 38.897 & 47.443 \\
PF50 & 22.833 & 6.6624 & 19.692 & 3.8329 & ---- & ---- \\
PF76 & 5.384 & 13.015 & 5.387 & 13.015 & 5.384 & 13.009 \\
\hline
\end{tabular}

Where, the maximum power flow in lines $34,40,50$ and 76 are 200, 150, 200 and $200 \mathrm{MW}$, respectively.

\section{- Unexpected outage of some units inside the generation plant}

Figures 3 and 4 show the SCOPD using ACO algorithm for different percentage outage of generation plants 1 and 3 for the 5-bus test system. From these figures, the power generation at bus 2 (PG2) is increased largely according to an increase in the percentage outage of power generations 1 and 3 . While, the power generations at buses 3 (PG3) and 1 (PG1) are increased smallly according to an increase in the percentage outage of power generations 1 and 3 , respectively shown in figures 3(a) and 4(a), as well as the generation costs are increased shown in figures 3(b) and 4(b).

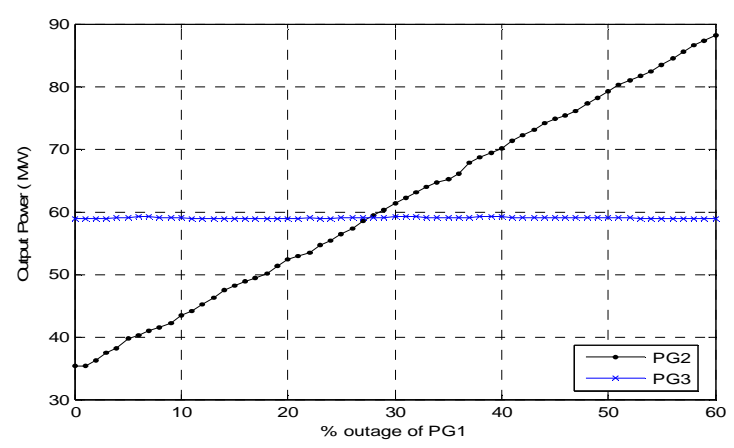

3. a) Variation of generation setting

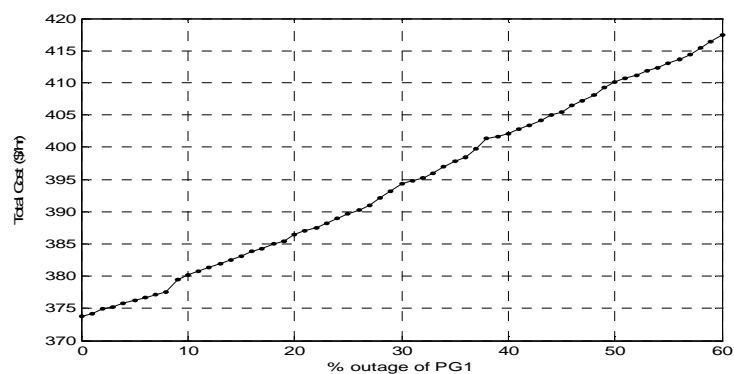

3. b) Variation of generation cost

Fig. 3 The SCOPD using ACO algorithm for different percentages outage of power generation 1

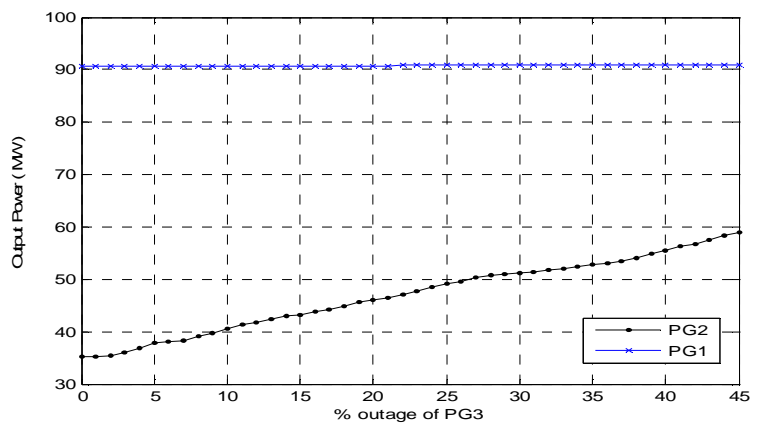

4. a) Variation of generation setting

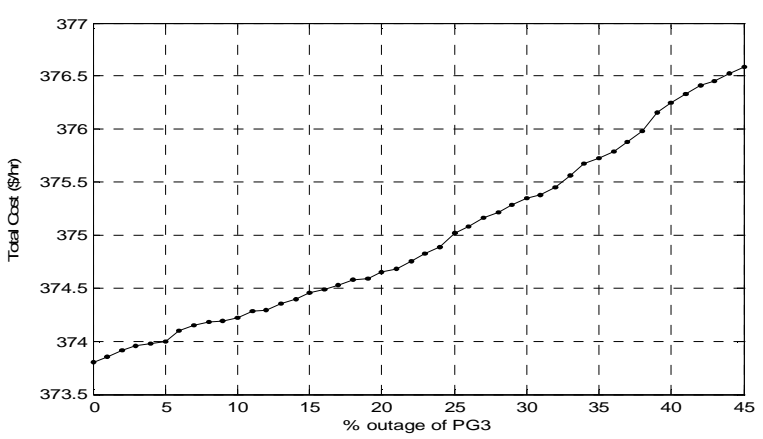

4. b) Variation of generation cost

Fig. 4 The SCOPD using ACO algorithm for different percentages outage of power generation 3 
Figure 5 shows the SCOPD using ACO algorithm for different percentage outage of generation plant 5 for the WDN system. From this figure, the power generation at other generation buses is increased according to an increase in the percentage outage of power generations 5, as well as the generation costs are increased.

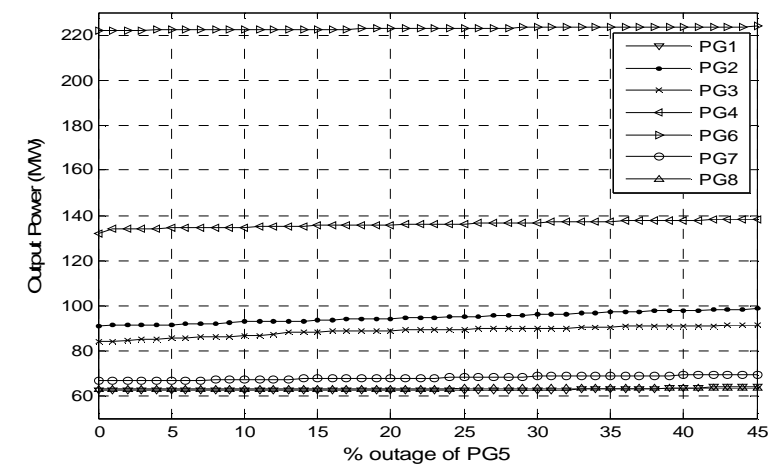

5. a) Variation of generation setting

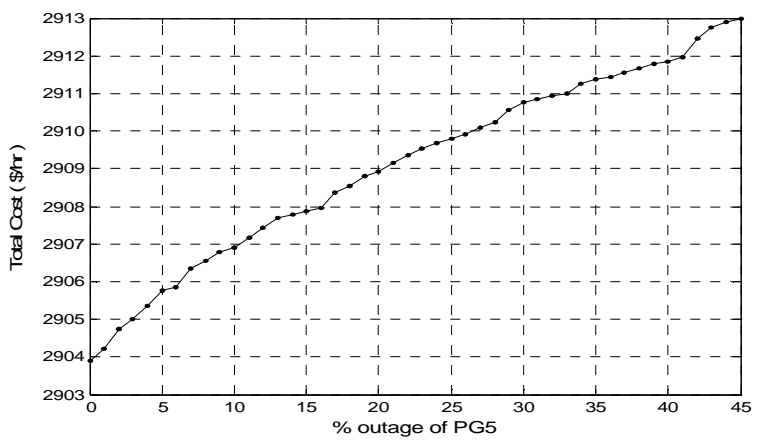

5. b) Variation of generation cost

Fig. 5 The SCOPD using ACO algorithm for different percentages outage of power generation 5

\section{CONCLUSION}

This paper presents an approach based on ACO algorithm to solve the problem of SCOPD with equality and inequality constraints under normal and emergency conditions. The proposed algorithm has been tested on a three test systems and real actual system is the WDN system as a part of the Unified Egyptian Network (UEN), the results obtained are compared with other conventional LP, FLP and GA. The results show that, ACO algorithm leads to minimum generation costs for normal condition, while all the power flows in the critical lines are kept within their permissible limits. So, the proposed ACO algorithm gives more accurate and efficiently solution to remove the insecure operation at different emergency conditions. Moreover, it needs a low memory in a PC because it is running in a decimal code unlike GA that running using a binary code. Therefore, the proposed algorithm represents a potential tool to aid the power system operators in the on-line environment.

\section{REFERENCES}

[1] M. Madrigal and Victor H. Quintana, "An Analytical Solution to the Economic Dispatch Problem", IEEE power Engineering Review, pp. 52-55, September 2000.

[2] Chun-Lung Chen and Nanming Chen, "Direct Search Method for Solving Economic Dispatch Problem Considering Transmission Capacity Constraints", IEEE Trans. on Power Systems, Vol. 16, No. 4, pp. 764-769, November 2001.

[3] Pathom Attaviriyanupap, Hiroyuki Kita, Eiichi Tanaka and Jun Hasegawa, "A Hybrid EP and SQP for Dynamic Economic Dispatch With Nonsmooth Fuel Cost Function", IEEE Trans. on Power Systems, Vol. 17, No. 2, pp. 411-416, May 2002.

[4] Qing Xia and Y. H. Song, "Dynamic Queuing Approach to Power System Short Term Economic and Security Dispatch", IEEE Trans. on Power Systems, Vol. 13, No. 2, pp. 280-285, May 1998.

[5] Xihui Yan and Victor H. Quintana, "Improving An Interior-Point-Based OPF by Dynamic Adjustments of Step Sizes and Tolerances", IEEE Trans. on Power Systems, Vol. 14, No. 2, pp. 709-717, May 1999.

[6] Rabih A. Jabr, Alun H. Coonick and Brian J. Cory, "A study of the Homogeneous Algorithm for Dynamic Economic Dispatch with Network Constraints and Transmission Losses", IEEE Trans. on Power Systems, Vol. 15, No. 2, pp. 605-611, May 2000.

[7] Lin Liu, Xifan Wang, Xiaoying Ding and Haoyong Chen, "A Robust Approach to Optimal Power Flow With Discrete Variables", IEEE Trans. on Power Systems, Vol. 24, No. 3, pp. 1182-1190, August 2009.

[8] Dragon Vlaisavljevic, Miodrag B. Djukanovic, Dejan J. Sobajic and Borivoje S. Babic, "Fuzzy Linear Programming Based Optimal Power System Rescheduling Including Preventive Redispatch", IEEE Trans. on Power Systems, Vol. 14, No. 2, pp. 525-531, May 1999.

[9] Pathom Attaviriyanupap, Hiroyuki Kita, Eiichi Tanaka and Jun Hasegawa, "A FuzzyOptimization Approach to Dynamic Economic Dispatch Considering Uncertainties", IEEE Trans. on Power Systems, Vol. 19, No. 3, pp. 1299-1307, August 2004.

[10]N. Amjady and H. Nasiri-Red, "Nonconvex Economic Dispatch With AC Constraints by a New Real Coded Genetic Algorithm", IEEE 
Trans. on Power Systems, Vol. 24, No. 3, pp. 1489-1502, August 2009.

[11]M. Dorigo, "Optimization, Learning and Natural Algorithms", Ph. D. dissertation, Dept. Electron., Politecnico di Milano, Milan, Italy, 1992.

[12]Dorigo M. and C. Blum, "Ant Colony Optimization Theory: A Survey", Theoretical Computer Science, Vol. 344 (2-3), pp. 243-278, 2005.

[13]Dorigo M. and L. M. Gambardella, "Ant Colonies for the Traveling Salesman Problem", BioSystems, Vol. 43, pp. 73-81, 1997.
[14] A. A. Abou El-Ela, M. A. Bishr, R. A. F. Saleh and T. Fetouh, "Security Constrained Optimal Dispatch Using Genetic Algorithms for Normal and Emergency Conditions", Engineering Research Journal (ERJ), Minoufiya University, Faculty of Engineering, Egypt, Vol. 28, No. 2, pp. 9-14, April 2005.

[15] Washington University Website: www.ee.washington.edu/research/pstca/

[16]R. A. El-Sehiemy, "Performance of Transmission Network Under Deregulated Electrical Power Systems", Ph. D. thesis, Minoufiya University, Faculty of Engineering, Egypt, August 2008. 